Rev. Biol. Trop., 48(2/3): 503-508, 2000

www.ucr.ac.cr www.ots.ac.cr www.ots.duke.edu

\title{
Hábitos alimentarios de las mojarras (Perciformes: Cichlidae) de la laguna Caobas, Quintana Roo, México
}

\author{
María Teresa Valtierra-Vega y Juan Jacobo Schmitter-Soto \\ El Colegio de la Frontera Sur (ECOSUR), Unidad Chetuma. Apdo. Postal 424, MX-77000 Chetumal, Q.R., México, \\ fax (983) 20447; teresa@ecosur-qroo.mx.
}

Recibido 10-VI-1999. Corregido 18-I-2000. Aceptado 4-II-2000.

\begin{abstract}
Feeding habits of seven cichlid species (Archocentrus octofasciatus, A. spilurus, "Cichlasoma" robertsoni, "C." synspilum, "C." urophthalmus, Petenia splendida, Thorichthys meeki) in Lake Caobas, southern Yucatan Peninsula, were studied. Samples were taken with enclosure and cast nets during the dry and rainy seasons of 1995 (day and night). The environment was characterized by measuring temperature, conductivity and $\mathrm{pH}$. All individuals were below $41 \mathrm{~mm} \mathrm{SL}(\mathrm{N}=281)$. Frequency of occurrence and prey abundance were analyzed. Main prey items were chironomids, mites, copepods, cladocerans, and ostracods. The cichlids fed mainly on zooplankton, with the partial exceptions of $P$. splendida (piscivore), "C." synspilum and A. spilurus (herbivores). A cluster analysis showed that the most similar trophic spectra were those of T. meeki, " $C$." robertsoni and " $C$." salvini, which were also the least diverse. " $C$." synspilum and $A$. spilurus had an intermediate distance between their diets and those of other species. The species with the most distinctive feeding composition were P. splendida (with the most diverse and equitable diet) and the omnivore A. octofasciatus (whose diet was the richest one). T. meeki showed quantitative diel, ontogenetic, and seasonal diet changes, but none between sexes. "C." robertsoni, "C." salvini and " $C$." synspilum differ in food habits in Caobas and in other localities, a fact that underscores the trophic adaptability of cichlids. Trophic overlap between cichlids in Caobas could imply absence of competition, perhaps because resources are abundant in the ecosystem.
\end{abstract}

Key words: Cichlidae, autoecology, feeding habits, Yucatán Peninsula, Mexico.

Los peces de la familia Cichlidae son conocidos por su capacidad de diversificación en función de la alimentación (Galls y Metz 1998). El resultado de esta diversificación puede ser la especiación explosiva, como la que se observa en los lagos africanos (Greenwood 1981), o bien un polimorfismo al interior de una sola especie, como en "Cichlasoma" minckleyi (Kornfield y Koehn 1975).

Por otro lado, también es usual encontrar en simpatría, e incluso sintopía, conjuntos de cíclidos de origen evolutivo diferente pero morfología similar. Por ejemplo, en la laguna de Bacalar, Quintana Roo, coexisten en los mismos hábitats ocho especies de mojarras
(Gamboa-Pérez y Schmitter-Soto 1998). Cabe preguntarse sobre los mecanismos de distribución de recursos de estos peces. En otros conjuntos ícticos, las diferencias en uso de hábitat son en primer lugar batimétrico, luego tróficas y por último alimentarias (Ross 1977).

El género Cichlasoma se restringe a Sudamérica (Kullander 1983). Algunas especies mexicanas antes incluidas en este taxon todavía no reciben una reasignación genérica definitiva, por lo que en este artículo han sido referidas a "Cichlasoma" (Miller y Norris 1996).

Entre los estudios sobre alimentación de varias especies de mojarras de la región están los de Chávez et al. (1988), Navarro-Mendoza 
(1988) y Martínez-Palacios y Ross (1994). Existen también trabajos sobre Thorichthys meeki (Neil 1984), "C." urophthalmus (CasoChávez et al. 1986, Martínez-Palacios y Ross 1988), "C." synspilum (Páramo 1987) y $P$. splendida (Reséndez-Medina y SalvadoresBaledón 1983).

El objetivo principal de este trabajo fue comparar entre sí los hábitos alimentarios de los cíclidos en la laguna Caobas, en términos de la composición, frecuencia y abundancia de las presas, variación ontogenética y por sexo, así como por periodo del día y por el mes.

\section{MATERIALES Y MÉTODOS}

La laguna Caobas se ubica en el sur del es-

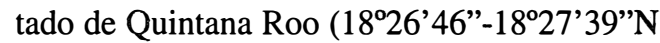
y $\left.89^{\circ} 04^{\prime} 03^{\prime \prime}-89^{\circ} 05^{\prime} 15^{\prime \prime} \mathrm{W}\right)$, a $100 \mathrm{~m}$ de altitud sobre el nivel del mar, unos $87 \mathrm{~km}$ al oeste de la ciudad de Chetumal. La circulación del agua de la laguna es primordialmente subterránea (Anónimo 1988). Está bordeada por selva mediana subperennifolia, un tular, vegetación secundaria arbórea, arbustiva y herbácea y zonas de cultivo seminómada (Anónimo 1985).

Las recolecciones se efectuaron en febrero, abril y julio de 1995. Con el objeto de caracterizar el ambiente acuático durante los muestreos, se midió en cada uno la temperatura del aire y del fondo de la laguna, conductividad y $\mathrm{pH}$, mediante aparatos portátiles. Las capturas se realizaron en muestreos diurnos y nocturnos utilizando chinchorro de $20 \times 1.5 \mathrm{~m}$, con $0.01 \mathrm{~cm}$ de abertura de malla, así como atarraya de $3 \mathrm{~m}$ de diámetro y $0.01 \mathrm{~cm}$ de luz de malla.

Los peces se fijaron con formol al $10 \%$. Luego se preservaron en etanol al $70 \%$ y se depositaron en la Colección de Peces de El Colegio de la Frontera Sur (ECOSUR), Unidad Chetumal) (acrónimo ECO-CH). A cada pez se le extrajo el tracto digestivo y se colocó en una caja de Petri. Los ítemes alimentarios se identificaron utilizando las claves de Pennak (1978) y Hanford y Edwin (1952).

Para estimar la abundancia relativa se midió el área ocupada por cada tipo de presa, una vez esparcido uniformemente el contenido estomacal en una caja de Petri cuadriculada en milímetros, con un área total de $2084 \mathrm{~mm}^{2}$. Bajo un microscopio estereoscópico, a $20 \mathrm{x}$ y $40 \mathrm{x}$, se observaron diez áreas de $16 \mathrm{~mm}^{2}$ escogidas de manera aleatoria. Los valores obtenidos se extrapolaron al área total. El conjunto de presas de cada especie se describió también mediante los índices de diversidad de Shannon (en nats/indiv.), riqueza de Margalef y equidad de Pielou (Magurran 1987). Para evaluar la variación ontogenética de la dieta, en las especies cuyo tamaño de muestra lo permitió, se compararon entre sí los peces maduros (estadios IV-V) e inmaduros (estadios I-III) (Valtierra y Pech 1997); asimismo, se efectuaron comparaciones por sexo, por hora y por mes, mediante el índice de Renkonen (Krebs 1989). Los cálculos se realizaron con ayuda de los programas Statistical Ecology (Ludwig y Reynolds 1988).

\section{RESULTADOS}

Durante el estudio, el pH varió de 6.55 a 8.23 (promedio, 6.99); la conductividad se mantuvo entre 280.0 y $287.7 \mu \mathrm{S} / \mathrm{cm}(294.4$ $\mu \mathrm{S} / \mathrm{cm}$ ) y la temperatura estuvo entre 25.1 y $29.3^{\circ} \mathrm{C}\left(27.7^{\circ} \mathrm{C}\right)$.

Se capturaron nueve especies de cíclidos, pero " $C$." friedrichsthali y " $C$." urophthalmus fueron escasas como para ser analizadas. Todos los individuos capturados fueron menores a $41 \mathrm{~mm}$ (longitud patrón). Se examinaron 281 tractos con alimento.

En los 127 tractos de T. meeki predominaron cladóceros y copépodos, tanto en abundancia como en frecuencia. Los copépodos fueron los harpacticoides Eucyclops agilis, Macrocyclops albidus y Mesocyclops longisetus.

En los 41 tractos de " $C$." robertsoni fueron frecuentes y abundantes los cladóceros, seguidos de quironómidos y copépodos. Los copépodos fueron $M$. edax, $M$. longisetus, $M$. reidae, Thermocyclops sp. y Tropocyclops prasinus.

En la alimentación de "C." salvini (37 tractos) fueron relevantes los cladóceros, copépodos 
(Macrocyclops albidus y Mesocyclops longisetus), quironómidos y ostrácodos. En A. octofasciatus (19 tractos) predominaron los ácaros, quironómidos, cladóceros y algas filamentosas. En el espectro trófico de "C." synspilum (31 tractos) las algas filamentosas fueron el ítem alimentario más abundante y éste fue el único cíclido que no consumió ácaros. En cuanto a $A$. spilurus (16 tractos), los ítemes alimentarios principales fueron algas filamentosas y ácaros. Por último, en Petenia splendida (18 tractos) los peces fueron la presa más frecuente, pero en términos de abundancia destacaron los cladóceros y ácaros.

Las especies tróficamente más semejantes entre sí fueron las zooplanctófagas $T$. meeki, "C." robertsoni y "C." salvini, cuyas dietas fueron además las menos diversas. Tampoco " $C$." synspilum y A. spilurus, las especies de tendencia herbívora, se distinguieron por su diversidad o la distancia media de su dieta respecto de las otras. Las especies de alimentación más diferente de las demás fueron la carnívora $P$. splendida (la de mayor diversidad y equidad de presas) y la omnívora A. octofasciatus (la de mayor riqueza alimentaria).

T. meeki fue la única especie cuyo volumen de captura permitió hacer comparaciones por sexo y ontogenéticas, así como por mes y periodo del día. El traslape de dietas entre machos y hembras fue superior al $80 \%$. Entre los individuos maduros y los inmaduros el traslape también fue elevado (76\%), pero varió la preferencia por algunas presas, en especial los quironómidos, que pasaron de una frecuencia del $12 \%$ en los tractos de peces inmaduros al $25 \%$ en los maduros, y las algas filamentosas, que subieron del 15 al $29 \%$.

El examen de las fluctuaciones de la dieta de T. meeki por mes reveló, entre otros cambios, que en marzo los cladóceros fueron el ítem alimentario más frecuente; los quironómidos y los peces, ausentes en febrero y marzo, tuvieron cierta importancia en mayo y julio; la frecuencia de los ostracodos decreció bruscamente en julio; y la hidra fue una presa encontrada sólo en dicho mes.
El traslape del espectro trófico de T. mee$k i$ entre los periodos diurno y nocturno fue apenas mayor al $50 \%$, debido a que la gran mayoría de las presas se ingirieron durante el día. Por la noche la dieta fue menos diversa, con predominio de peces $(40 \%)$ y anélidos (20\%); además, las hidras sólo se encontraron en el período nocturno.

\section{DISCUSIÓN}

Con base en este estudio, T. meeki se considera zooplanctófago; A. octofasciatus, omnívoro; A. spilurus, herbívoro con tendencia zooplanctófaga, y $P$. splendida, carnívoro. Los mismos resultados se reportan en la literatura (Reséndez-Medina y Salvadores-Baledón 1983, Neil 1984, Chávez et al. 1986, Páramo 1987, Navarro-Mendoza 1988, Martínez-Palacios y Ross 1994).

Un rasgo que distingue parcialmente el espectro trófico de $P$. splendida en Caobas es la tendencia zooplanctófaga, en comparación con la franca piscivoría detectada por los autores mencionados. Es posible que lo pequeño de las tallas capturadas en Caobas sean la causa de la importancia relativamente menor de los peces en la alimentación local de esta especie, y que el cambio a una alimentación piscívora ocurra más adelante en el desarrollo ontogenético, cuando el tamaño de la boca facilite ese tipo de depredación. Este proceso es usual en muchas especies ícticas (Schmitter-Soto y Castro-Aguirre 1996) y tiene la ventaja evolutiva de evitar la competencia intraespecífica entre adultos y juveniles.

Los espectros tróficos de las otras tres especies, especialmente el de " $C$." robertsoni, fueron menos coincidentes con los datos previos (Cuadro 1). Estas diferencias en la composición del espectro alimentario de una especie en diferentes regiones corroboran la adaptabilidad de los Cichlidae. Tampoco se descartan fluctuaciones en las preferencias tróficas de una especie dentro de una misma zona en diferentes años (Sierra et al. 1994). 


\section{CUADRO 1}

Comparación entre las tendencias tróficas de algunos cíclidos según este estudio y según otros autores.

TABLE 1

Comparison between trophic tendencies of some cichlids according to this study and other authors.

$\begin{array}{llll}\text { Especie } & \begin{array}{l}\text { Tendencia trófica } \\ \text { (en Caobas) }\end{array} & \begin{array}{l}\text { Tendencia trófica } \\ \text { (otros autores) }\end{array} & \text { Autores } \\ \text { "C." robertsoni } & \text { zooplanctófago } & \begin{array}{l}\text { herbívoro } \\ \text { carnívoro }\end{array} & \begin{array}{l}\text { MP\&R } \\ \text { NM }\end{array} \\ \text { "C." salvini } & \text { zooplanctófago } & \text { carnívoro } & \text { MP\&R } \\ \text { "C." synspilum } & \text { herbívoro-omnívoro } & \begin{array}{l}\text { herbívoro-omnívoro } \\ \text { omnívoro herbívoro } \\ \text { herbívoro }\end{array} & \begin{array}{l}\text { MP\&R } \\ \mathrm{C} \& *\end{array} \\ & & \text { NM, PD }\end{array}$

*Cháves et al. (1983) identificaron a "C" synspilum como "C." Fenestrarum.

PD, Páramo 1987, en las lagunas Santa Anita y Horizonte, Tabasco; NM, Navarro-Mendoza 1988, en cenotes de Sian Ka'an, Quintana Roo; MP\&R, Martínez-Palacios y Ross 1994, localidades sin precisar.

PD, Páramo 1987, in lakes Santa Anita and Horizonte, Tabasco; NM, Navarro-Mendoza 1988, in cenotes of Sian Ka'an, Quintana Roo; MP\&R, Martínez-P 1994, unspecified localitie

Además de que se observa un gran traslape trófico entre todas las especies (así como entre los sexos y estados de madurez de T. meeki), todas ellas pueden catalogarse como generalistas, no sólo por su diversidad trófica, sino también porque se alimentan tanto en la columna de agua como en la superficie y en el fondo (como lo demuestra la ingestión de copépodos bénticos, epibénticos y pláncticos, así como ácaros de la superficie, por un mismo individuo).

Esta aparente falta de competencia puede atribuirse a que los recursos alimentarios son abundantes en el ecosistema. Tampoco Gamboa-Pérez y Schmitter-Soto (1998) encontraron un reparto de hábitat evidente entre los cíclidos de la laguna de Bacalar, todos los cuales prefieren fondos blandos, con abundante vegetación y materia orgánica. Esta tendencia de los cíclidos fue observada también en la laguna de Términos (ReséndezMedina 1981), a pesar de exhibir ésta un ambiente muy distinto.

\section{AGRADECIMIENTOS}

El trabajo fue parte de la tesis de licenciatura de la primera autora y fue apoyado parcialmente por el Consejo Nacional de Ciencia y Tecnología de México (CONACYT), a través del proyecto 1829-N. Eduardo Suárez-Morales proporcionó la identificación y hábitat de los copépodos. Scott Monks confirmó que los nẹmátodos eran parásitos, no presas.

\section{RESUMEN}

Se estudió la alimentación de siete especies de cíclidos (Archocentrus octofasciatus, A. spilurus, "Cichlasoma" robertsoni, " $C$." synspilum, " $C$." urophthalmus, $P$ tenia splendida, Thorichthys meeki) en la laguna Caobas, sur de la península de Yucatán. Los muestreos se llevaron a cabo durante las estaciones seca y lluviosa de 1995. Los ejemplares medían menos de $41 \mathrm{~mm}$ LP. Se evaluó la frecuencia de aparición y la abundancia de las presas. Los ítemes alimentarios predominantes fueron los quironómidos, ácaros, copépodos, cladóceros y ostrácodos. La mayoría de las mojarras se mostraron zooplanctófagas, con la 
excepción parcial de $P$. splendida (piscívora), " $C$." synspilum y A. spilurus (herbívoras). A. octofasciatus tuvo el más amplio espectro alimentario y se consideró omnívoro. Thorichthys meeki mostró cambios cuantitativos en la dieta por hora del día, talla del pez y estación del año, no así por sexo.

\section{REFERENCIAS}

Anónimo. 1985. Carta del uso del suelo y vegetación. 1:250 000. Inst. Nac. Estadíst. Geogr. Informát., México.

Anónimo. 1988. Carta hidrológica de aguas superficiales. 1: 250000. Inst. Nac. Estadíst. Geogr. Informát., México.

Caso-Chávez, M., A. Yáñez-Arancibia \& A. L. Lara-Domínguez. 1986. Biology, ecology and population dynamics of Cichlasoma urophthalmus (Günther) en hábitat de Thalassia testudinum y Rhizophora mangle, Laguna de Términos, sur del Golfo de México. Biótica 11: 79-111.

Chávez L., M.O., A.E. Mattheeuws \& M.H. Pérez V. 1988. Biología de los peces del río San Pedro en vista de determinar su potencial para la piscicultura. Inst. Nac. Invest. Recursos Bióticos, Villahermosa, México. 222 p.

Galls, F. \& J.A.J. Metz. 1998. Why are there so many cichlid species? Trends Ecol. Evolut. 13: 1-2.

Gamboa-Pérez, H.C. \& J.J. Schmitter-Soto. 1998. Distribution of cichlid fishes in Lake Bacalar, Yucatan Peninsula. Env. Biol. Fishes 54: 35-43.

Greenwood, P.H. 1981. Species-flocks and explosive evolution, p. 61-74. In The evolving biosphere. Museo Británico, Londres.

Hanford, T.L. \& M. Edwin. 1952. The algae of Illinois. Hafner, Chicago.

Kornfield, I.L. \& R.K. Koehn. 1975. Genetic variation and speciation in New World cichlids. Evolution 29: 427-437.

Krebs, J.C. 1989. Ecological methodology. Harper \& Row, Nueva York.

Kullander, S. 1983. A revision of the South American cichlid genus Cichlasoma (Teleostei: Cichlidae). Museo Sueco de Historia Natural, Estocolmo.

Ludwig, A.J. \& F.J. Reynolds. 1988. Statistical ecology: A primer on methods and computing. Wiley, Nueva York.
Magurran, A.E. 1987. Ecological diversity and its measurement. Princeton, Nueva Jersey.

Martínez-Palacios, C.A. \& L.G. Ross. 1988. The feeding ecology of the Central American cichlid Cichlasoma urophthalmus (Gunther). J. Fish Biol. 33: 665-670.

Martínez-Palacios, C.A. \& L.G. Ross. 1994. Biología y cultivo de la mojarra latinoamericana: Cichlasoma urophthalmus. Consejo Nacional de Ciencia y Tecnología/Centro de Investigación para la Alimentación y el Desarrollo, México. 203 p.

Miller, R.R. \& S.M. Norris. 1996. A progress report on the higher classifications of Middle American cichlids. Abstr. 76 ${ }^{\text {th }}$ Meet. Amer. Soc. Ichthyol. Herpetol., Nueva Orleans: 219.

Navarro-Mendoza, M. 1988. Inventario íctico y estudios ecológicos preliminares de los cuerpos de agua continentales en la reserva de la biosfera de Sian Ka'an y áreas circunvecinas en Quintana Roo, México. Centro de Investigaciones de Quintana Roo/Secretaría de Desarrollo Urbano y Ecología/ United States Fish \& Wildlife Service, Chetumal, México.

Neil, S. 1984. Field studies of the behavioral ecology and agonistic behavior of Cichlasoma meeki (Pisces: Cichlidae) Env. Biol. Fishes 10: 59-68.

Páramo D., S. 1987. Algunos aspectos biológicos de la mojarra Cichlasoma synspilum en las lagunas Santa Anita y Horizonte, Tab., Méx. Resúms. IX Congr. Nac. Zool., Villahermosa, México, No. 37.

Pennak, W.R. 1978. Fresh-water invertebrates of the United States. Ronald, Nueva York.

Reséndez-Medina, A. 1981. Estudio de los peces de la laguna de Términos, Campeche, México. I-II. Biótica 6: 239-291, 345-430.

Reséndez-Medina, A. \& M.L. Salvadores-Baledón. 1983. Contribución al conocimiento de la biología del pejelagarto Lepisosteus tropicus (Gill) y la tenguayaca Petenia splendida Günther, del estado de Tabasco. Biótica 8: 413-426.

Ross, S.T. 1977. Patterns of resource partitioning in searobins (Pisces: Triglidae). Copeia 1977: 561-571.

Schmitter-Soto, J.J. \& J.L. Castro-Aguirre. 1996. Trophic comparison among Triglidae (Pisces: Scorpaeniformes) off Baja California Sur, Mexico. Rev. Biol. Trop. 44: 803-811. 
Sierra M., L., R. Claro \& O. Popova. 1994. Alimentación y relaciones tróficas, p. 263-286. In R. Claro (Ed.). Ecología de los peces marinos de Cuba. Centro de Invetigación de Quintana Roo, Chetumal, México. ValtierraV., M.T. \&
U. Pech R. 1997. Aspectos tróficos y reproductivos de las mojarras (Teleostei: Cichlidae) de la laguna Caobas, Quintana Roo. Tesis de Licenciatura, Universidad Michoacana de San Nicolás de Hidalgo, Morelia, México. 